\title{
Measurement of Loudness Discomfort Levels as a Test for Hyperacusis: Test-Retest Reliability and Its Clinical Value
}

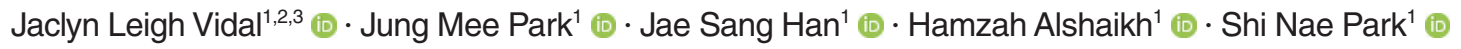 \\ ${ }^{I}$ Department of Otorhinolaryngology-Head and Neck Surgery, Seoul St. Mary's Hospital, College of Medicine, The Catholic University of Korea, \\ Seoul, Korea; ${ }^{2}$ Department of Otorhinolaryngology-Head and Neck Surgery, Dr. Paulino J. Garcia Memorial Research and Medical Center, \\ Nueva Ecija; ${ }^{3}$ Department of Otorhinolaryngology-Head and Neck Surgery, St. Luke's Medical Center, Quezon City, Philippines
}

Objectives. The aims of this study were to investigate the test-retest reliability of measurements of loudness discomfort levels (LDLs), to suggest cut-off values for diagnosing patients with hyperacusis, and to evaluate the clinical value of LDL measurements as a test for monitoring hyperacusis.

Methods. For the test-retest reliability of LDL measurements (study 1), a total of 68 patients who sought consultations at our clinic were subcategorized into four groups: patients with tinnitus (group 1), tinnitus and hearing loss (group 2), hyperacusis (group 3), and normal controls (group 4). Inter-hour and inter-day test-retest reliability values using different stimuli were investigated. For study 2 , the clinical value of LDL measurements using pure tone stimuli was analyzed by comparing changes after sound generator use in patients with hyperacusis.

Results. In study 1, the group 3 patients showed significantly lower LDLs than the other groups. High test-retest reliability of LDL tests was demonstrated, regardless of the type of stimulus used. The cut-off values for screening patients with hyperacusis were $90 \mathrm{~dB}$ HL using pure tone stimuli and $62 \mathrm{~dB}$ HL using white-band noise stimuli. In study 2, significantly increased LDLs were correlated with improved symptoms and improved scores on tinnitus questionnaires after sound generator use, indicating that LDL measurement is a reliable test for monitoring hyperacusis during an intervention.

Conclusion. LDL measurement is a reliable diagnostic tool to reflect the condition of hyperacusis, especially during the course of treatment.

Keywords. Hyperacusis; Loudness Perception; Sound Generator

\section{INTRODUCTION}

Hyperacusis is characterized by decreased sound tolerance or auditory hypersensitivity to ordinary environmental sounds [1].

\footnotetext{
- Received February 18, 2021

Revised July 4, 2021

Accepted July 14, 2021

- Corresponding author: Shi Nae Park

Department of Otorhinolaryngology-Head and Neck Surgery, Seoul St.

Mary's Hospital, College of Medicine, The Catholic University of Korea,

222 Banpo-daero, Seocho-gu, Seoul 06591, Korea

Tel: +82-2-2258-6215, Fax: +82-2-595-1354

E-mail: snparkmd@catholic.ac.kr
}

A person with hyperacusis may have an exaggerated response or discomfort to sounds that are normally non-aversive to a typical person [2,3]. Hyperacusis has been described using several terms such as phonophobia, misophonia, and recruitment. These terms, however, have substantial differences. Phonophobia is the fear of episodic sound intolerance, which is brought about by excessive stimulation of the autonomic and limbic systems. Misophonia is a developed aversion to sounds such as chewing or breathing.

The diagnosis of hyperacusis remains obscure since there is no gold-standard objective test that can accurately determine the presence of hyperacusis in a person [4]. Most studies have de-

Copyright $@ 2022$ by Korean Society of Otorhinolaryngology-Head and Neck Surgery.

This is an open-access article distributed under the terms of the Creative Commons Attribution Non-Commercial License (https://creativecommons.org/licenses/by-nc/4.0)

which permits unrestricted non-commercial use, distribution, and reproduction in any medium, provided the original work is properly cited. 
scribed diagnosing hyperacusis based on a self-report questionnaire called the Hyperacusis Questionnaire formulated by Khalfa et al. [5] This hyperacusis questionnaire was found to be beneficial for the quantification and characterization of the clinical phenomenon of hyperacusis over three major dimensions: attentional, social, and emotional [6].

Several methods have been used in attempts to measure hyperacusis objectively. The assessment of hyperacusis usually involves pure tone audiometry (PTA), acoustic reflex test (ART), tone decay test, otoacoustic emissions (OAE), auditory brainstem response (ABR), and loudness discomfort levels (LDLs). Since hyperacusis is primarily characterized by an individual's abnormal loudness perception, it is logical that an individual's loudness discomfort perception is measured. Hence, this study focused on the measurement of LDLs.

LDLs predict the level at which tones are detected by a person to be uncomfortably loud. LDLs have been commonly used to quantify hyperacusis, but their reliability as a diagnostic tool has not yet actually been proven. The average LDL of normal-hearing individuals is approximately $100 \mathrm{~dB}$ HL [7]. The average LDL of hyperacusis patients varies across studies because the criteria for a strict diagnosis of hyperacusis are not generally agreed upon. The study of Goldstein and Shulman [8] suggested that an LDL less than $95 \mathrm{~dB} \mathrm{HL}$ is an indication of hyperacusis, while it was $70 \mathrm{~dB}$ HL according to Anari et al. [9], and 80, 85, 80, and 75 dB HL at 500, 1,000, 2,000, and 4,000 Hz, respectively, according to Sherlock and Formby [7]. This variability of LDL values across studies is, therefore, a major limitation of LDL measurements [10].

Hence, the first part of this study aimed to investigate the testretest reliability of LDL measurements in the diagnosis of hyperacusis. After establishing the reliability of the test, we can determine whether it is actually a good measure of hyperacusis. We also aimed to suggest cut-off values for diagnosing hyperacusis. In the second part of the study, we aimed to evaluate the clinical value of LDL measurements by monitoring LDL changes in patients with hyperacusis who were prescribed sound generators. The use of sound generators in the treatment of hyperacusis has been promising [11-13]. It would therefore be interesting to evaluate whether the efficacy of sound generators for patients with hyperacusis is reflected in their LDLs.

\section{H I G H L I G H T S}

- The retest reliability of loudness discomfort level (LDL) measurements was generally excellent.

- LDL measurements for hyperacusis had high test specificity.

- A significant increase in LDL values was seen in patients who used sound generators.

\section{MATERIALS AND METHODS}

\section{Subject selection and evaluation}

This study was conducted on patients who sought consult at the outpatient clinic of a tertiary referral center.

\section{Institutional approval}

Informed consent was obtained from the participants, and approval was obtained from the Institutional Review Board of Seoul St. Mary's Hospital approved this study (IRB No. KC17RESI0422).

\section{Study 1: test-retest reliability of LDLs}

Data of 68 patients of tinnitus and hyperacusis clinic were used for the first study. Patients were asked to fill up questionnaires upon their initial consult. They were then categorized into four groups based on their chief complaint: patients with only tinnitus (group 1), tinnitus and hearing loss (group 2), patients with only hyperacusis (group 3) and normal control (group 4). Subjective patients with hyperacusis (group 3) were selected and defined as the patients complaining of increased sound sensitivity to usual environmental sound confirmed by positivity in both hyperacusis questionnaire and an interview by tinnitus specialist.

Their LDLs were measured using pure tone $(125-8,000 \mathrm{~Hz})$ and white band noise by $5 \mathrm{~dB}$ and $2 \mathrm{~dB}$ ascending method respectively. Duration of each stimulus was one second. First hyperacusis test/LDL measurement was done during the patient's initial visit. Second LDL measurement (inter-day measurement) was on his second visit, which was 1 week after the first visit. Third measurement (inter-hour measurement) was 1 hour after the second measurement. Their LDLs were compared with normal controls to suggest the cut-off point for hyperacusis.

\section{Study 2: clinical value of LDLs}

For the second study, the clinical value of the LDLs using pure tone stimuli were analyzed by comparing the changes of LDL values before and after sound generator use in 100 patients with tinnitus. Patients were grouped into two. Group A comprised of patients who only had tinnitus, while group $\mathrm{B}$ are patients who had hyperacusis and tinnitus.

Initial assessment of hyperacusis was performed by asking the patients to answer the following question at the initial visitAre you particularly sensitive to or bothered by noise or certain environmental sounds? A hyperacusis questionnaire was also used to evaluate the patient's condition of hyperacusis. To detect changes of hyperacusis symptoms, the patients were asked to rate how much the symptoms of hyperacusis troubled them in their daily life on a scale of 10 , at every visit during their treatment period. Patients were also asked to answer questionnaires on visual analog scale (VAS) scores of subjective tinnitus distress (loudness/awareness/annoyance/effect on life) and Tinnitus Handicap Inventory (THI).

Patients were recommended to use sound generators if there 
is failure of response to environmental sound therapy or if there is distressing hyperacusis. The patients' LDL values were then measured initially and after 6 months of sound generator use. Patients were instructed to use sound generators for at least 8 hours a day. The intensity of the sound from sound generators were from low to high up to mixing point, which indicated the sound level changing patients' tinnitus.

\section{Statistical analysis}

All data for study 1 were entered into SAS ver. 6.12 (SAS Institute Inc., Cary, NC, USA) to assess the reliability within or intersubjects using repeated analysis of variance (ANOVA) test. Cutoff values were suggested by receiver operating characteristic (ROC) curve in SAS logit analysis. Mean LDLs among groups were compared by ANOVA test, and $P$-value less than 0.05 was considered as statistically significant. The statistical analysis for study 2 was performed using IBM SPSS ver. 21.0 (IBM Corp., Armonk, NY, USA) at a significance level of 0.05 .

Table 1. Clinical characteristics of the patients in study 1

\begin{tabular}{llcc}
\hline Group & Age $(y r)$ & Sex $(M: F)$ & Hearing threshold ${ }^{a)}(d B H L)$ \\
\hline $1(n=15)$ & $46.9 \pm 13.6$ & $9: 6$ & $19.1 \pm 8.1$ \\
$2(n=12)$ & $55.9 \pm 7.9$ & $4: 8$ & $56.3 \pm 23.3$ \\
$3(n=21)$ & $47.9 \pm 11.0$ & $10: 11$ & $25.7 \pm 13.3$ \\
$4(n=20)$ & $31.6 \pm 9.3$ & $10: 10$ & $13.9 \pm 8.8$ \\
\hline
\end{tabular}

Values are presented as mean \pm standard deviation. Group 1, patients with tinnitus; group 2, patients with tinnitus and hearing loss; group 3, patients with hyperacusis; group 4, normal control.

a) Mean air conduction hearing level at 500, 1,000, 2,000, and 3,000 Hz.

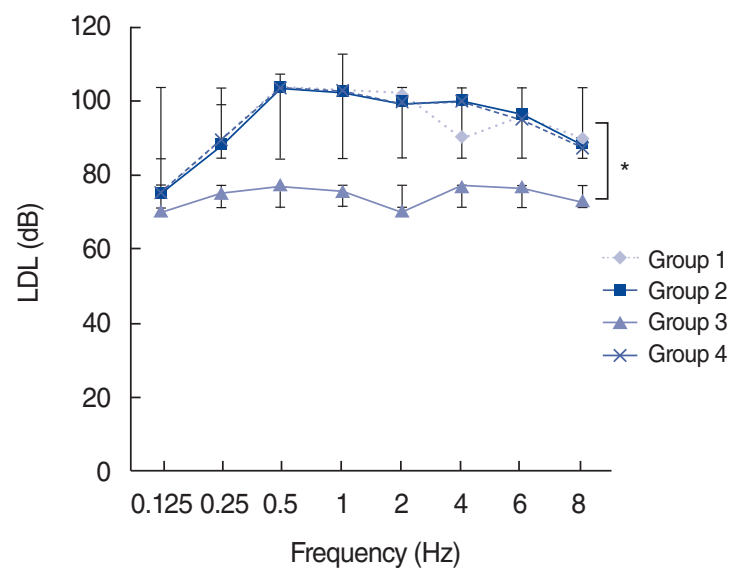

Fig. 1. Mean loudness discomfort levels (LDLs) using pure tone stimuli at different frequencies, showing that the patients in group 3 had lower mean LDLs (74 dB; standard deviation [SD], 2.96). Group 1 $(n=15)$ : patients with tinnitus; group $2(n=12)$ : patients with tinnitus and hearing loss; group $3(n=21)$ : patients with hyperacusis; group $4(n=20)$ : normal control. ${ }^{*} P<0.05$. Error bars indicate SDs. Tests of the hypotheses were assessed using the $t$-test for six independent groups per frequency with a Bonferroni-adjusted alpha level of 0.008 per test $(0.05 / 6)$.

\section{RESULTS}

\section{Study 1: test-retest reliability}

The clinical characteristics of the patients involved in the first study are summarized in Table 1 . There were four groups in study 1: group 1 comprised 15 patients with only tinnitus ( 9 men, 6 women; mean age, 46.9 years; standard deviation [SD], 13.6 years); group 2 comprised 12 patients with tinnitus and hearing impairment, with an average threshold of pure tone audiogram in speech frequencies over $25 \mathrm{~dB} \mathrm{HL}$ (mean, $56.3 \pm 23.3 \mathrm{~dB} \mathrm{HL}$; 4 men, 8 women; mean age, 55.9 years; SD, 7.9 years); group 3 comprised 21 patients with hyperacusis (10 men, 11 women; mean age, 47.9 years; SD, 11 years), and group 4 comprised 20 patients in the normal control group (10 men, 10 women; mean age, 31.6 years; SD, 9.3 years).

The mean LDL curve of group 3 using pure tone stimuli was significantly lower $(74 \mathrm{~dB}$; $\mathrm{SD}, 2.96 \mathrm{~dB})$ than that of the other groups for all frequencies (group 1: $94 \mathrm{~dB}, \mathrm{SD}, 9.92 \mathrm{~dB}$; group 2: $94 \mathrm{~dB}, \mathrm{SD}, 9.67 \mathrm{~dB}$; group 4: $93.9 \mathrm{~dB}, \mathrm{SD}, 9.52 \mathrm{~dB} ; P<0.05$ ) (Fig. 1). The mean LDL value using white-band noise stimuli was $45.1 \mathrm{~dB}(\mathrm{SD}, 12.1 \mathrm{~dB})$ for group 3 , which was significantly lower than the mean LDLs of the other non-hyperacusic groups (group 1: $73.9 \mathrm{~dB}$, group 2: $70.9 \mathrm{~dB}$, and group 4: $73.8 \mathrm{~dB} ; P<0.01$ ) (Fig. 2). The inter-hour and inter-day reliability coefficients of the hyperacusis test using pure tone stimuli in all of the subjects were relatively high $(r=0.8-0.93)$ except at $0.25 \mathrm{kHz}(r=0.66)$ (Fig. 3). The reliability coefficients of the hyperacusis test using whiteband noise were as high as 0.95 and 0.93 for the inter-hour and inter-day tests, respectively.

The sensitivity and specificity of the cut-off values for hyper-

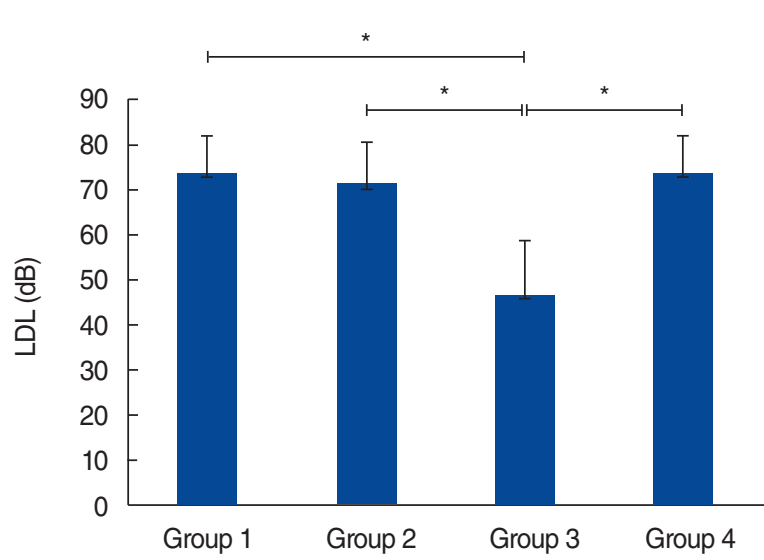

Fig. 2. Mean loudness discomfort levels (LDLs) using white-band noise, showing that group 3 patients had statistically significantly lower mean LDL values, at $45.1 \mathrm{~dB} \mathrm{HL}$ (standard deviation [SD], $12.1 \mathrm{~dB} \mathrm{HL}, P<0.01$. Group $1(n=15)$ : patients with tinnitus; group 2 $(n=12)$ : patients with tinnitus and hearing loss; group $3(n=21)$ : patients with hyperacusis; and group $4(n=20)$ : normal control patients. ${ }^{\star} P<0.05$. Error bars indicate SDs. Tests of the hypotheses were assessed using the $t$-test for six independent groups with a Bonferroni adjusted alpha level of 0.008 per test $(0.05 / 6)$. 
acusis tests according to pure tone and white-band noise stimuli are shown in Table 2. For pure tone stimuli, the cut-off values suggested by the ROC curve analysis were from 70 to $90 \mathrm{~dB}$. The cut-off value of $90 \mathrm{~dB}$ from 500 to $8 \mathrm{kHz}$ showed high specificity $(94.4 \%-99.2 \%)$ but relatively low sensitivity $(70.4 \%-83.2 \%)$. For white-band noise, the cut-off value suggested was $62 \mathrm{~dB}$, which had high specificity (93.7\%) and low sensitivity (73.5\%).

\section{Study 2: clinical value of LDL measurements}

Two groups of tinnitus patients with or without subjective hyperacusis were enrolled in study 2. Group A included patients

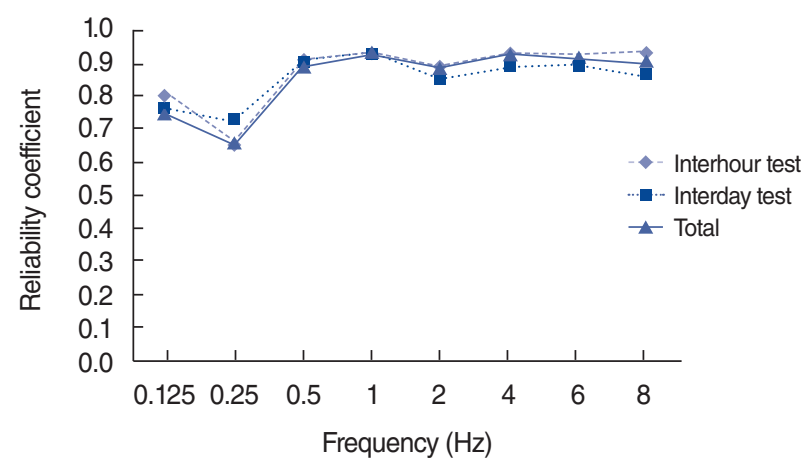

Fig. 3. Inter-hour and inter-day reliability coefficients of loudness discomfort level measurements using pure-tone stimuli $(r=0.8-0.93)$.

Table 2. Suggested cut-off values with corresponding sensitivity and specificity values using receiver operating characteristic curve analysis (study 1)

\begin{tabular}{lcccccccccc}
\hline \multirow{2}{*}{$\begin{array}{c}\text { Stimulus } \\
\text { frequency }\end{array}$} & \multicolumn{8}{c}{ Pure tone (Frequency, Hz) } & WBN \\
\cline { 2 - 10 } & 0.125 & 0.25 & 0.5 & 1 & 2 & 4 & 6 & 8 & \\
\hline Cut-off (dB) & 70 & 85 & 90 & 90 & 90 & 90 & 90 & 90 & 62 \\
Sensitivity (\%) & 95.1 & 80.3 & 80.9 & 75.3 & 72.8 & 79.1 & 70.4 & 83.2 & 73.5 \\
Specificity (\%) & 56.4 & 90.5 & 94.4 & 97.6 & 98.4 & 94.4 & 97.6 & 99.2 & 93.7 \\
\hline
\end{tabular}

WBN, white-band noise. who had only tinnitus, while group B included patients who had tinnitus and hyperacusis. There were 40 men and 24 women in group A, with a mean age of 49.5 years (SD, 13.22 years). Group $B$ had 11 men and 25 women, with a mean age of 49.4 years (SD, 13.52 years) (Table 3). The patients in group B showed lower mean average initial LDLs $(97.9 \mathrm{~dB})$ than those in group A (102.8 dB). However, this difference was not statistically significant $(P=0.08$, Student $t$-test).

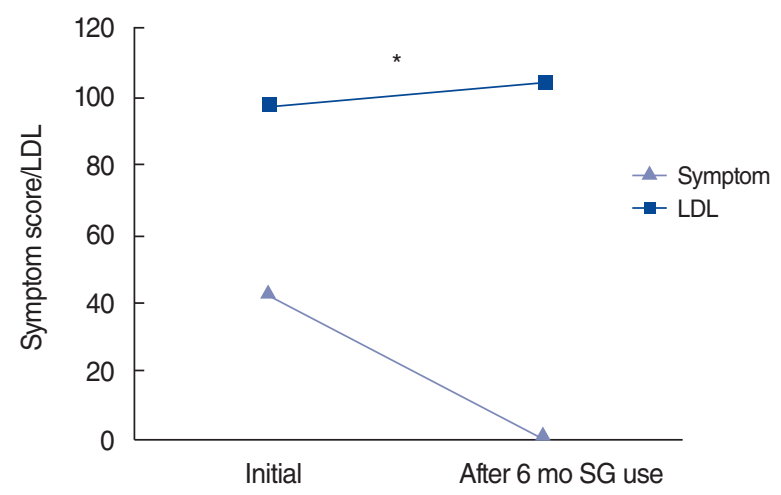

Fig. 4. Comparison of hyperacusis symptoms and loudness discomfort level (LDL) measurements in group 2 patients. Significantly increased LDLs and decreased symptoms of hyperacusis were observed in the tinnitus patients with hyperacusis after 6 months of sound generator (SG) use $\left({ }^{\star} P=0.02\right)$.

Table 3. Clinical characteristics of the patients in study 2

\begin{tabular}{lccc}
\hline Variable & Group A $(n=64)$ & Group B $(n=36)$ & $P$-value \\
\hline Sex (male:female) & $40: 24$ & $11: 25$ & 0.041 \\
Age $(y r)$ & $49.5 \pm 13.2$ & $49.4 \pm 13.5$ & 0.972 \\
Average PTA ${ }^{\text {a) }}(\mathrm{dB} \mathrm{HL})$ & $20.8 \pm 12.5$ & $19.1 \pm 15.6$ & 0.514 \\
\hline
\end{tabular}

Values are presented as mean \pm standard deviation. Group A, patients with tinnitus without hyperacusis; group B, patients with tinnitus and hyperacusis

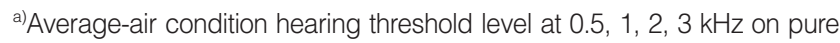
tone audiograms, in $\mathrm{dB} \mathrm{HL}$ ( $\mathrm{dB}$ hearing level).

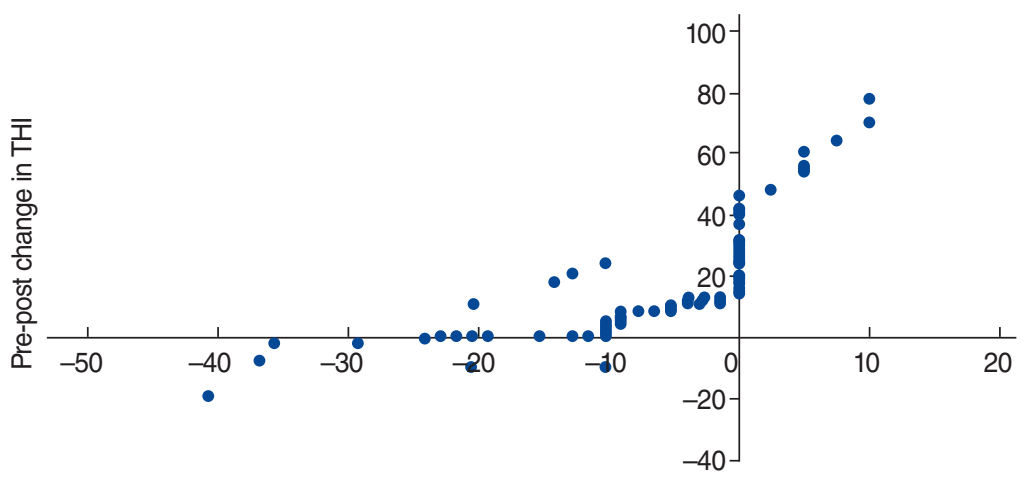

Pre-post change in LDL

Fig. 5. Correlations between mean loudness discomfort level (LDL) values and patients' scores on the Tinnitus Handicap Inventory (THI). Scatter plot of pre-post changes in the THI versus pre-post changes in LDLs, showing that a larger decrease in THI scores was correlated with a larger increase in LDLs $(r=0.73, P<0.05)$. 
After 6 months of sound generator use, the patients in group B claimed that their subjective hyperacusis considerably improved. Upon comparison of the subjective symptoms and objective LDL measurements of the group B patients, a significant decrease was found in their subjective hyperacusis symptoms with a corresponding increase in their average LDL after 6 months of sound generator use $(P=0.02)$ (Fig. 4).

An inverse correlation between the patients' LDL values and their scores on the tinnitus questionnaires was also noted, with $r$-values from -0.73 to -0.92 , indicating a strong association (THI, -0.88 ; loudness, -0.92 ; awareness, -0.86 ; annoyance, -0.86 ; and effect on life, -0.85$)$. Patients' THI scores seemed to decrease as their LDL thresholds increased, as seen in Fig. 5 ( $r=0.73$, $P<0.05)$.

\section{DISCUSSION}

Hyperacusis is a condition wherein a person has abnormally strong reactions after exposure to sounds that are low to moderate in intensity, which leads to decreased tolerance to suprathreshold sounds $[14,15]$. Hyperacusis is said to be a precursor of tinnitus, and about $40 \%$ of patients with tinnitus also experience hyperacusis $[16,17]$.

It is important to differentiate hyperacusis from the development of the loudness recruitment phenomenon. A person who experiences the loudness recruitment phenomenon most likely has cochlear hearing loss and dysfunction of the outer hair cells of the organ of Corti. The individual's perceived loudness increases faster than normal upon exposure to an increasing intensity of sound in loudness recruitment. There is also narrowing of the dynamic range caused by the higher detection threshold, and the discomfort level is unaffected [18]. This is in contrast with hyperacusis, where an individual's perceived loudness increases even in response to sounds of low to moderate intensity. There is usually no associated hearing loss, as was demonstrated in studies 1 and 2, since the patients enrolled in both studies had normal hearing thresholds (Tables 1 and 3).

The diagnosis of hyperacusis usually relies on the functional handicap or effect on life produced by this symptom. The handicap associated with hyperacusis can be quantified using the instrument published by Khalfa et al. [5], which is a self-report questionnaire with 14 items focusing on three factors (attentional, social, and emotional). The Multiple Activity Scale for Hyperacusis is another questionnaire used to evaluate the effect of hyperacusis on individuals' daily living [19].

These are, nonetheless, only subjective measures of hyperacusis. There is still no uniform standardized diagnostic procedure for hyperacusis. Some researchers have attempted to measure hyperacusis objectively by using PTA, ART, the tone decay test, OAE, ABR, and LDLs. These tests, however, measure different areas in the auditory pathway, which would then perpetuate the question of how hyperacusis develops in the first place. It is therefore important to determine the pathogenesis of hyperacusis to find out whether it is quantifiable by a single diagnostic test.

Several potential mechanisms have been proposed in attempts to explain hyperacusis. One mechanism is rooted in the observation that both hyperacusis and tinnitus worsen during episodes of anxiety, stress, or fatigue. Endogenous dynorphins are said to be released during times of stress. These dynorphins are released into the synaptic regions of hair cells, and cause the release of glutamate, which is known to influence the intensity tuning of auditory processing. This, in turn, causes sound stimuli to be perceived as louder than they should be [20]. Auditory efferent dysfunction is another possible mechanism of hyperacusis. This mechanism is based on the problem of central gain modulation, which causes sensitivity to sound [21,22].

Another well-referenced mechanism is the neurophysiological model suggested by Jastreboff et al. [23]. This model postulates that the limbic system, autonomic nervous system, and auditory systems are all activated when a person has hyperacusis and tinnitus. There is central hyperexcitability in the central auditory system $[20,23]$. This central hyperexcitability is a key point supporting the possibility that a diagnostic test measuring the central auditory system, such as LDL measurements, would be a more appropriate objective measure of hyperacusis than other diagnostic tests. In this study, we focused on evaluating LDL measurements in patients with hyperacusis.

For study 1, inter-hour and inter-day testing of LDLs using two different types of stimuli were done. Two different types of stimuli were used because patients with hyperacusis usually complain of discomfort when exposed to certain environmental sounds. Pure tone stimuli only have a narrow frequency spectrum; hence, the additional use of white-band noise can increase the spectrum to which patients must respond. The retest reliability of LDL measurements using pure tone stimuli and white-band noise was generally excellent. The reliability values were higher than 0.9 using white-band noise and pure tone stimuli. The high retest reliability of LDL indicates that the patients' LDLs were consistent even across different days and different hours in the day.The good test-retest reliability of LDL measurement in this study may be attributed to the type of patients included. In particular, group 3 comprised patients who solely complained of hyperacusis, unlike previous studies focusing on patients who had both hyperacusis and tinnitus [10]. Another factor that may have contributed to the high test-retest reliability of LDLs in our study seems to be proper instruction and the fact that the pure tone test signal was given to the study subjects by a single specialized audiologist, which minimized the measurement error caused by the tester.

Initially, cut-off values were determined in study 1 to predict the exact level at which hyperacusis can be diagnosed. Based on the mean hyperacusis response values in study 1 , the cut-off values derived were 90 and $62 \mathrm{~dB}$ for pure tone and white-band 
noise stimuli, respectively. These cut-off values showed high specificity, but low sensitivity. A screening test with good specificity has the ability to correctly identify those without the disease. Test sensitivity, in contrast, is the ability to correctly identify those with the disease. The high specificity and low sensitivity of our derived cut-off values imply that LDL measurement may not be effective for the initial screening of patients with hyperacusis. LDL measurement may instead be more beneficial in ruling out those without hyperacusis and in monitoring patients' progress/ improvement when given a particular intervention. Hence, LDL use is more beneficial when measuring the effectiveness of a particular treatment. For the initial diagnosis, it is advisable to use LDLs in conjunction with other validated questionnaires to accurately determine whether a patient actually has hyperacusis. It is also important to mention that the cut-off values suggested are particularly useful for patients complaining of hyperacusis alone. For patients complaining of both hyperacusis and tinnitus, a higher cut-off value may be recommended in future studies.

Since the results of the first study showed that LDL measurements are suitable for monitoring hyperacusis during treatment, we incorporated our initial findings and used LDL measurements to monitor the progress of patients with hyperacusis and tinnitus upon prescription of sound generators. Sound generators, when given in addition to counseling, have been shown to be effective for the treatment of patients with hyperacusis $[1,12,13]$. Desensitization is the main concept of sound therapy [3].

Using this concept, study 2 involved prescribing sound generators to patients who had distressing hyperacusis or did not respond to environmental sound therapy. An increase in the LDL value after sound generator use would mean that there was an increase in the threshold of discomfort experienced by the patient. The results of study 2 showed that there was a significant increase in LDL values in patients who used sound generators. This objective improvement in LDL values was seen to be associated with the subjective improvement of the patients' hyperacusic symptoms (Fig. 4). The improvement was also reflected in patients' responses to questionnaires: the THI and VAS scores for loudness, awareness, annoyance, and effect on life. As their LDL values increased, their scores on the questionnaires decreased, showing a strong and significant inverse correlation. There was a greater improvement in their THI scores as their LDL thresholds increased (Fig. 5).

Conflicting reports have been published regarding the relationship between LDLs and patients' self-reported hyperacusis handicap [24]. Some studies reported a weak negative correlation between across-frequency average LDLs and self-reported sound tolerance problems [25]. Some studies did not find a statistically significant relationship [19], while others reported a moderate and highly significant negative correlation between self-reports of hyperacusis and LDLs [26]. In our study, there was a strong inverse correlation between LDLs and patients' scores on the questionnaires, meaning that measurements of LDLs indeed reflected the hyperacusic component of the subjective complaints of tinnitus.

The patients who complained of hyperacusis in study 2 showed a mean LDL value of $97 \mathrm{~dB}$, which was slightly higher than the suggested cut-off value in study 1 , which was $90 \mathrm{~dB}$. This difference might be explained by the presence of comorbid tinnitus in the patients in study 2 . In study 1 , the patients in group 3 complained of hyperacusis only, while in study 2 , the patients in group $\mathrm{B}$ complained of tinnitus and comorbid hyperacusis. Therefore, the patients with only hyperacusis in study 1 seemed to have lower LDLs than the hyperacusis patients with tinnitus in study 2.This may indicate that the good sensitivity, specificity, and testretest reliability of LDL measurements are particularly useful for patients who complain of hyperacusis only.

Based on the results of study 1 and study 2, LDL measurements are important in the stepwise approach to hyperacusis. The results of study 1 show that LDL measurements are a reliable tool for monitoring hyperacusis. The good test-retest reliability signifies the internal validity of the test and that the measurements obtained are both representative and stable over time. The cut-off values for diagnosis, $90 \mathrm{~dB}$ for pure tone stimuli and $62 \mathrm{~dB}$ for white band noise, had a relatively low sensitivity but high specificity, which indicates the clinical value of LDL measurements as a diagnostic test for determining patients' progress during an intervention rather than as a screening test. After being diagnosed with hyperacusis, patients can be monitored for improvement in response to an intervention. The objective improvement in patients' LDL can also have a positive effect as they can objectively see that they are increasing their threshold for uncomfortable sounds, as was clearly demonstrated in study 2 .

In conclusion, LDL measurements have high inter-day and inter-hour reliability regardless of the type of stimulus used (pure tone or white-band noise), indicating that this test has good internal validity. LDL measurements are particularly reliable in patients who complain of hyperacusis alone but are also helpful in those who have comorbid tinnitus. Significantly increased LDLs and decreased hyperacusis symptoms were observed in tinnitus patients with hyperacusis after 6 months of sound generator use. A study analyzing the correlation of scores on hyperacusis questionnaires and LDL scores can be done in the future to further strengthen the results of this study. LDL measurement is a reliable and valuable diagnostic tool to reflect the condition of hyperacusis during the course of an intervention.

\section{CONFLICT OF INTEREST}

No potential conflict of interest relevant to this article was reported. 


\section{ACKNOWLEDGMENTS}

This research was supported by the Basic Science Research Program through the National Research Foundation of Korea (NRF) funded by the Ministry of Education (2018R1D1A1A02048972).

\section{ORCID}

$\begin{array}{ll}\text { Jaclyn Leigh Vidal } & \text { https://orcid.org/0000-0002-0699-1592 } \\ \text { Jung Mee Park } & \text { https://orcid.org/0000-0001-8589-4119 } \\ \text { Jae Sang Han } & \text { https://orcid.org/0000-0001-7728-1232 } \\ \text { Hamzah Al Shaikh } & \text { https://orcid.org/0000-0003-3634-9821 } \\ \text { Shi Nae Park } & \text { https://orcid.org/0000-0002-7614-9413 }\end{array}$

\section{AUTHOR CONTRIBUTIONS}

Conceptualization: SNP. Data curation: JLV, JMP, SNP. Formal analysis: JLV, JMP, JSH, SNP. Funding acquisition: SNP. Methodology: JLV, JSH, HAS. Project administration: JLV, SNP. Visualization: SNP.Writing-original draft: JLV.Writing-review \& editing: JLV, SNP.

\section{REFERENCES}

1. Fackrell K, Potgieter I, Shekhawat GS, Baguley DM, Sereda M, Hoare DJ. Clinical interventions for hyperacusis in adults: a scoping review to assess the current position and determine priorities for research. Biomed Res Int. 2017;2017:2723715.

2. Aazh H, Moore BC, Lammaing K, Cropley M. Tinnitus and hyperacusis therapy in a UK National Health Service audiology department: patients' evaluations of the effectiveness of treatments. Int $\mathrm{J}$ Audiol. 2016 Sep;55(9):514-22.

3. Baguley DM. Hyperacusis. J R Soc Med. 2003 Dec;96(12):582-5.

4. Yilmaz S, Tas M, Bulut E, Nurcin E. Assessment of reduced tolerance to sound (hyperacusis) in university students. Noise Health. 2017 Mar-Apr;19(87):73-8.

5. Khalfa S, Dubal S, Veuillet E, Perez-Diaz F, Jouvent R, Collet L. Psychometric normalization of a hyperacusis questionnaire. ORL J Otorhinolaryngol Relat Spec. 2002 Nov-Dec;64(6):436-42.

6. Greenberg B, Carlos M. Psychometric properties and factor structure of a new scale to measure hyperacusis: introducing the inventory of hyperacusis symptoms. Ear Hear. 2018 Sep/Oct;39(5):1025-34.

7. Sherlock LP, Formby C. Estimates of loudness, loudness discomfort, and the auditory dynamic range: normative estimates, comparison of procedures, and test-retest reliability. J Am Acad Audiol. 2005 Feb;
16(2):85-100.

8. Goldstein B, Shulman A.Tinnitus: hyperacusis and the loudness discomfort level test- a preliminary report. Int Tinnitus J. 1996;2:83-9.

9. Anari M, Axelsson A, Eliasson A, Magnusson L. Hypersensitivity to sound: questionnaire data, audiometry and classification. Scand Audiol. 1999;28(4):219-30.

10. Baguley DM, Hoare DJ. Hyperacusis: major research questions. HNO. 2018 May;66(5):358-63.

11. Pienkowski M. Rationale and efficacy of sound therapies for tinnitus and hyperacusis. Neuroscience. 2019 May;407:120-34.

12. Park JM, Kim WJ, Ha JB, Han JJ, Park SY, Park SN. Effect of sound generator on tinnitus and hyperacusis. Acta Otolaryngol. 2018 Feb; 138(2):135-9.

13. Sheppard A, Stocking C, Ralli M, Salvi R. A review of auditory gain, low-level noise and sound therapy for tinnitus and hyperacusis. Int $\mathrm{J}$ Audiol. 2020 Jan;59(1):5-15.

14. Aazh H, McFerran D, Moore BC. Uncomfortable loudness levels among children and adolescents seeking help for tinnitus and/or hyperacusis. Int J Audiol. 2018 Aug;57(8):618-23.

15. Suhnan AP, Finch PM, Drummond PD. Hyperacusis in chronic pain: neural interactions between the auditory and nociceptive systems. Int J Audiol. 2017 Nov;56(11):801-9.

16. Wallen MB, Hasson D, TheorellT, Canlon B.The correlation between the hyperacusis questionnaire and uncomfortable loudness levels is dependent on emotional exhaustion. Int J Audiol. 2012 Oct;51(10): 722-9.

17. Schecklmann M, Landgrebe M, Langguth B; TRI Database Study Group. Phenotypic characteristics of hyperacusis in tinnitus. PLoS One. 2014 Jan;9(1):e86944.

18. Katzenell U, Segal S. Hyperacusis: review and clinical guidelines. Otol Neurotol. 2001 May;22(3):321-6.

19. Meeus OM, Spaepen M, Ridder DD, Heyning PH. Correlation between hyperacusis measurements in daily ENT practice. Int J Audiol. 2010 Jan;49(1):7-13.

20. Sahley TL, Nodar RH. A biochemical model of peripheral tinnitus. Hear Res. 2001 Feb;152(1-2):43-54.

21. Auerbach BD, Radziwon K, Salvi R. Testing the central gain model: loudness growth correlates with central auditory gain enhancement in a rodent model of hyperacusis. Neuroscience. 2019 May;407:93107.

22. Sheldrake J, Diehl PU, Schaette R. Audiometric characteristics of hyperacusis patients. Front Neurol. 2015 May;6:105.

23. Jastreboff PJ, Gray WC, Gold SL. Neurophysiological approach to tinnitus patients. Am J Otol. 1996 Mar;17(2):236-40.

24. Aazh H, Moore BC. Factors related to uncomfortable loudness levels for patients seen in a tinnitus and hyperacusis clinic. Int J Audiol. 2017 Oct;56(10):793-800.

25. Zaugg TL, Thielman EJ, Griest S, Henry JA. Subjective reports of trouble tolerating sound in daily life versus loudness discomfort levels. Am J Audiol. 2016 Dec;25(4):359-63.

26. Blaesing L, Kroener-Herwig B. Self-reported and behavioral sound avoidance in tinnitus and hyperacusis subjects, and association with anxiety ratings. Int J Audiol. 2012 Aug;51(8):611-7. 